\title{
A DEGREE THEORY FOR A CLASS OF PERTURBED FREDHOLM MAPS II
}

\author{
PIERLUIGI BENEVIERI, ALESSANDRO CALAMAI, AND MASSIMO FURI
}

Received 30 June 2005; Revised 10 October 2005; Accepted 24 October 2005

In a recent paper we gave a notion of degree for a class of perturbations of nonlinear Fredholm maps of index zero between real infinite dimensional Banach spaces. Our purpose here is to extend that notion in order to include the degree introduced by Nussbaum for local $\alpha$-condensing perturbations of the identity, as well as the degree for locally compact perturbations of Fredholm maps of index zero recently defined by the first and third authors.

Copyright (c) 2006 Pierluigi Benevieri et al. This is an open access article distributed under the Creative Commons Attribution License, which permits unrestricted use, distribution, and reproduction in any medium, provided the original work is properly cited.

\section{Introduction}

In a recent paper [1] we defined a concept of degree for a special class of noncompact perturbations of nonlinear Fredholm maps of index zero between (infinite dimensional real) Banach spaces, called $\alpha$-Fredholm maps. The definition of these maps is based on the following two numbers (see, e.g., [12]) associated with a map $f: \Omega \rightarrow F$ from an open subset of a Banach space $E$ to a Banach space $F$ :

$$
\begin{aligned}
& \alpha(f)=\sup \left\{\frac{\alpha(f(A))}{\alpha(A)}: A \subseteq \Omega \text { bounded, } \alpha(A)>0\right\}, \\
& \omega(f)=\inf \left\{\frac{\alpha(f(A))}{\alpha(A)}: A \subseteq \Omega \text { bounded, } \alpha(A)>0\right\},
\end{aligned}
$$

where $\alpha$ is the Kuratowski measure of noncompactness (in [12] $\omega(f)$ is denoted by $\beta(f)$, however, we prefer here the more recent notation $\omega(f)$ as in [9]).

Roughly speaking, an $\alpha$-Fredholm map is of the type $f=g-k$, with the inequality

$$
\alpha(k)<\omega(g)
$$

satisfied locally. These maps include locally compact perturbations of Fredholm maps (quasi-Fredholm maps for short) since, when $g$ is Fredholm and $k$ is locally compact,

Hindawi Publishing Corporation

Fixed Point Theory and Applications

Volume 2006, Article ID 27154, Pages 1-20

DOI 10.1155/FPTA/2006/27154 
one has $\alpha(k)=0$ and $\omega(g)>0$, locally. Moreover, they also contain local $\alpha$-contractive perturbations of the identity, where, following Darbo [6], a map $k$ is $\alpha$-contractive if $\alpha(k)<1$.

The purpose of this paper is to give an extension of the notion of the degree for $\alpha$ Fredholm maps to a more general class of noncompact perturbations of Fredholm maps, still defined in terms of the numbers $\alpha$ and $\omega$. This class of maps, that we call weakly $\alpha$-Fredholm, includes local $\alpha$-condensing perturbations of the identity, where a map $k$ is $\alpha$-condensing if $\alpha(k(A))<\alpha(A)$, for every $A$ such that $0<\alpha(A)<+\infty$. We show that, for local $\alpha$-condensing perturbations of the identity, our degree coincides with the degree defined by Nussbaum in $[14,15]$.

For an interesting, although partial, extension of the Leray-Schauder degree to a large class of maps (called quasi-ruled Fredholm maps) we mention the work of Efendiev (see $[10,11]$ and references therein). This class of maps has nonempty intersection with our class of weakly $\alpha$-Fredholm maps. However, our degree is integer valued and, as said before, extends completely the Nussbaum degree (and, consequently, the Leray-Schauder degree). This is not the case of the degree by Efendiev, since it takes values in the nonnegative integers.

\section{Orientability for Fredholm maps}

In this section we summarize the notion of orientability for nonlinear Fredholm maps of index zero between Banach spaces introduced in [2,3].

The starting point is a concept of orientation for linear Fredholm operators of index zero between real Banach spaces. From now on and in the rest of the paper, $E$ and $F$ will denote two real Banach spaces. Recall that a bounded linear operator $L: E \rightarrow F$ is said to be Fredholm if $\operatorname{dim} \operatorname{Ker} L$ and $\operatorname{dim} \operatorname{coKer} L$ are finite. The index of $L$ is

$$
\text { ind } L=\operatorname{dim} \operatorname{Ker} L-\operatorname{dim} \operatorname{coKer} L \text {. }
$$

Given a Fredholm operator of index zero $L: E \rightarrow F$, a bounded linear operator $A: E \rightarrow$ $F$ with finite dimensional image is called a corrector of $L$ if $L+A$ is an isomorphism. On the (nonempty) set $\mathscr{C}(L)$ of correctors of $L$ we define an equivalence relation as follows. Let $A, B \in \mathscr{C}(L)$ be given and consider the following automorphism of $E$ :

$$
T=(L+B)^{-1}(L+A)=I-(L+B)^{-1}(B-A) .
$$

The operator $K=(L+B)^{-1}(B-A)$ clearly has finite dimensional image. Hence, given any nontrivial finite dimensional subspace $E_{0}$ of $E$ containing the image of $K$, the restriction of $T$ to $E_{0}$ is an automorphism. Therefore, its determinant is well defined and nonzero. It is easy to check that this does not depend on the choice of $E_{0}$ (see [2]). Thus, the determinant of $T$ is well defined as the determinant of the restriction of $T$ to any nontrivial finite dimensional subspace of $E$ containing the image of $K$. We say that $A$ is equivalent to $B$ or, more precisely, $A$ is L-equivalent to $B$ if

$$
\operatorname{det}\left((L+B)^{-1}(L+A)\right)>0 \text {. }
$$


As shown in [2], this is actually an equivalence relation on $\mathscr{C}(L)$ with two equivalence classes.

Definition 2.1. Let $L$ be a linear Fredholm operator of index zero between two real Banach spaces. An orientation of $L$ is the choice of one of the two equivalence classes of $\mathscr{C}(L)$, and $L$ is oriented when an orientation is chosen.

Given an oriented operator $L$, the elements of its orientation are called positive correctors of $L$.

Definition 2.2. An oriented isomorphism $L$ is said to be naturally oriented if the trivial operator is a positive corrector, and this orientation is called the natural orientation of $L$.

An orientation of a Fredholm operator of index zero induces an orientation to any sufficiently close operator. Precisely, consider a Fredholm operator of index zero $L$ and a corrector $A$ of $L$. Since the set of the isomorphisms from $E$ into $F$ is open in the space $L(E, F)$ of bounded linear operators, $A$ turns out to be a corrector of every $T$ in a suitable neighborhood $U$ of $L$ in $L(E, F)$. Therefore, if $L$ is oriented and $A$ is a positive corrector of $L$, any $T \in U$ can be oriented taking $A$ as a positive corrector of $T$. This fact allows us to give a notion of orientation for a continuous map with values in the set $\Phi_{0}(E, F)$ of Fredholm operators of index zero from $E$ into $F$.

Definition 2.3. Let $X$ be a topological space and $h: X \rightarrow \Phi_{0}(E, F)$ a continuous map. An orientation of $h$ is a continuous choice of an orientation $\alpha(x)$ of $h(x)$ for each $x \in X$, where "continuous" means that for any $x \in X$ there exists $A \in \alpha(x)$ which is a positive corrector of $h\left(x^{\prime}\right)$ for any $x^{\prime}$ in a neighborhood of $x$. A map is orientable when it admits an orientation and oriented when an orientation is chosen.

Remark 2.4. It is possible to prove (see [3, Proposition 3.4]) that two equivalent correctors $A$ and $B$ of a given $L \in \Phi_{0}(E, F)$ remain $T$-equivalent for any $T$ in a neighborhood of $L$. This implies that the notion of "continuous choice of an orientation" in Definition 2.3 is equivalent to the following one:

(i) for any $x \in X$ and any $A \in \alpha(x)$, there exists a neighborhood $U$ of $x$ such that $A \in \alpha\left(x^{\prime}\right)$ for all $x^{\prime} \in U$.

As a straightforward consequence of Definition 2.3, if $h: X \rightarrow \Phi_{0}(E, F)$ is orientable and $g: Y \rightarrow X$ is any continuous map, then the composition $h g$ is orientable as well. In particular, if $h$ is oriented, then $h g$ inherits in a natural way an orientation from the orientation of $h$. This holds, for example, for the restriction of $h$ to any subset $A$ of $X$, since $\left.h\right|_{A}$ is the composition of $h$ with the inclusion $A \hookrightarrow X$. Moreover, if $H: X \times[0,1] \rightarrow$ $\Phi_{0}(E, F)$ is an oriented homotopy and $\lambda \in[0,1]$ is given, the partial map $H_{\lambda}=H i_{\lambda}$, where $i_{\lambda}(x)=(x, \lambda)$, inherits an orientation from $H$.

The following proposition shows an important property of the notion of orientability for continuous maps in $\Phi_{0}(E, F)$, which is, roughly speaking, a sort of continuous transport of an orientation along a homotopy (see [3, Theorem 3.14]).

Proposition 2.5. Consider a homotopy $H: X \times[0,1] \rightarrow \Phi_{0}(E, F)$. Assume that, for some $\lambda \in[0,1]$, the partial map $H_{\lambda}=H(\cdot, \lambda)$ is oriented. Then there exists a unique orientation of $H$ such that the orientation of $H_{\lambda}$ is inherited from that of $H$. 
Let us now give a notion of orientability for Fredholm maps of index zero between Banach spaces. Recall that, given an open subset $\Omega$ of $E$, a map $g: \Omega \rightarrow F$ is a Fredholm map if it is $C^{1}$ and its Fréchet derivative, $g^{\prime}(x)$, is a Fredholm operator for all $x \in \Omega$. The index of $g$ at $x$ is the index of $g^{\prime}(x)$ and $g$ is said to be of index $n$ if it is of index $n$ at any point of its domain.

Definition 2.6. An orientation of a Fredholm map of index zero $g: \Omega \rightarrow F$ is an orientation of the continuous map $g^{\prime}: x \mapsto g^{\prime}(x)$, and $g$ is orientable, or oriented, if so is $g^{\prime}$ according to Definition 2.3.

The notion of orientability of Fredholm maps of index zero is discussed in depth in $[2,3]$, where the reader can find examples of orientable and nonorientable maps. Here we recall a property (Theorem 2.8 below) which is the analogue for Fredholm maps of the continuous transport of an orientation along a homotopy, as seen in Proposition 2.5. We need first the following definition.

Definition 2.7. Let $H: \Omega \times[0,1] \rightarrow F$ be a $C^{1}$ homotopy. Assume that any partial map $H_{\lambda}$ is Fredholm of index zero. An orientation of $H$ is an orientation of the map

$$
\partial_{1} H: \Omega \times[0,1] \longrightarrow \Phi_{0}(E, F), \quad(x, \lambda) \longmapsto\left(H_{\lambda}\right)^{\prime}(x)
$$

and $H$ is orientable, or oriented, if so is $\partial_{1} H$ according to Definition 2.3.

From the above definition it follows immediately that if $H$ oriented, an orientation of any partial map $H_{\lambda}$ is inherited from $H$.

Theorem 2.8 below is a straightforward consequence of Proposition 2.5.

Theorem 2.8. Let $H: \Omega \times[0,1] \rightarrow F$ be $C^{1}$ and assume that any $H_{\lambda}$ is a Fredholm map of index zero. If a given $H_{\lambda}$ is orientable, then $H$ is orientable. If, in addition, $H_{\lambda}$ is oriented, there exists a unique orientation of $H$ such that the orientation of $H_{\lambda}$ is inherited from that of $H$.

We conclude this section by showing that the orientation of a Fredholm map $g$ is related to the orientations of domain and codomain of suitable restrictions of $g$. This argument will be crucial in the definition of the degree for quasi-Fredholm maps.

Let $g: \Omega \rightarrow F$ be an oriented map and $Z$ a finite dimensional subspace of $F$, transverse to $g$. By classical transversality results, $M=g^{-1}(Z)$ is a differentiable manifold of the same dimension as $Z$. In addition, $M$ is orientable (see [2, Remark 2.5 and Lemma 3.1]). In particular, let us show how, given any $x \in M$, the orientation of $g$ and a chosen orientation of $Z$ induce an orientation on the tangent space $T_{x} M$ of $M$ at $x$.

Let $Z$ be oriented. Consider $x \in M$ and a positive corrector $A$ of $g^{\prime}(x)$ with image contained in $Z$ (the existence of such a corrector is ensured by the transversality of $Z$ to $g$ ). Then, orient $T_{x} M$ in such a way that the isomorphism

$$
\left.\left(g^{\prime}(x)+A\right)\right|_{T_{x} M}: T_{x} M \longrightarrow Z
$$

is orientation preserving. As proved in [4], the orientation of $T_{x} M$ does not depend on the choice of the positive corrector $A$, but only on the orientations of $Z$ and $g^{\prime}(x)$. With this orientation, we call $M$ the oriented $g$-preimage of $Z$. 


\section{Orientability and degree for quasi-Fredholm maps}

In this section we recall the concept of degree for quasi-Fredholm maps. This degree was defined for the first time in [16] by means of the Elworthy-Tromba notion of Fredholm structure on a differentiable manifold. Here we summarize the simple approach given in [4] which is based on the concept of orientation for nonlinear Fredholm maps and avoids the Elworthy-Tromba theory.

The starting point is the definition of orientability for quasi-Fredholm maps.

Definition 3.1. Let $\Omega$ be an open subset of $E, g: \Omega \rightarrow F$ a Fredholm map of index zero and $k: \Omega \rightarrow F$ a locally compact map. The map $f: \Omega \rightarrow F$, defined by $f=g-k$, is called a quasi-Fredholm map and $g$ is a smoothing map of $f$.

The following definition provides an extension to quasi-Fredholm maps of the concept of orientability.

Definition 3.2. A quasi-Fredholm map $f: \Omega \rightarrow F$ is orientable if it has an orientable smoothing map.

If $f$ is an orientable quasi-Fredholm map, any smoothing map of $f$ is orientable. Indeed, given two smoothing maps $g_{0}$ and $g_{1}$ of $f$, consider the homotopy $H: \Omega \times[0,1] \rightarrow$ $F$, defined by

$$
H(x, \lambda)=(1-\lambda) g_{0}(x)+\lambda g_{1}(x)
$$

Notice that any $H_{\lambda}$ is Fredholm of index zero, since it differs from $g_{0}$ by a $C^{1}$ locally compact map. By Theorem 2.8, if $g_{0}$ is orientable, then $g_{1}$ is orientable as well.

Let $f: \Omega \rightarrow F$ be an orientable quasi-Fredholm map. To define a notion of orientation of $f$, consider the set $\mathscr{Y}(f)$ of the oriented smoothing maps of $f$. We introduce in $\mathscr{P}(f)$ the following equivalence relation. Given $g_{0}, g_{1}$ in $\mathscr{S}(f)$, consider, as in formula (3.1), the straight-line homotopy $H$ joining $g_{0}$ and $g_{1}$. We say that $g_{0}$ is equivalent to $g_{1}$ if their orientations are inherited from the same orientation of $H$, whose existence is ensured by Theorem 2.8. It is immediate to verify that this is an equivalence relation. If the domain of $f$ is connected, any smoothing map has two orientations and, hence, $\mathscr{S}(f)$ has exactly two equivalence classes.

Definition 3.3. Let $f: \Omega \rightarrow F$ be an orientable quasi-Fredholm map. An orientation of $f$ is the choice of an equivalence class in $\mathscr{Y}(f)$.

By the above construction, given an orientable quasi-Fredholm map $f$, an orientation of a smoothing map $g$ determines uniquely an orientation of $f$. Therefore, in the sequel, if $f$ is oriented, we will refer to a positively oriented smoothing map of $f$ as an element in the chosen class of $\mathscr{S}(f)$.

As for Fredholm maps of index zero, the orientation of quasi-Fredholm maps verifies a homotopy invariance property, as shown in Theorem 3.6 below. We need first some definitions. 
6 A degree theory for a class of perturbed Fredholm maps II

Definition 3.4. Let $H: \Omega \times[0,1] \rightarrow F$ be a map of the form

$$
H(x, \lambda)=G(x, \lambda)-K(x, \lambda)
$$

where $G$ is $C^{1}$, any $G_{\lambda}$ is Fredholm of index zero and $K$ is locally compact. We call $H$ a homotopy of quasi-Fredholm maps and $G$ a smoothing homotopy of $H$.

We need a concept of orientability for homotopies of quasi-Fredholm maps. The definition is analogous to that given for quasi-Fredholm maps. Let $H: \Omega \times[0,1] \rightarrow F$ be a homotopy of quasi-Fredholm maps. Let $\mathscr{S}(H)$ be the set of oriented smoothing homotopies of $H$. Assume that $\mathscr{S}(H)$ is nonempty and define on this set an equivalence relation as follows. Given $G_{0}$ and $G_{1}$ in $\mathscr{S}(H)$, consider the map

$$
\mathscr{H}: \Omega \times[0,1] \times[0,1] \longrightarrow F
$$

defined as

$$
\mathscr{H}(x, \lambda, s)=(1-s) G_{0}(x, \lambda)+s G_{1}(x, \lambda) .
$$

We say that $G_{0}$ is equivalent to $G_{1}$ if their orientations are inherited from an orientation of the map

$$
(x, \lambda, s) \longmapsto \partial_{1} \mathscr{H}(x, \lambda, s)
$$

The reader can easily verify that this is actually an equivalence relation on $\mathscr{S}(H)$.

Definition 3.5. A homotopy of quasi-Fredholm maps $H: \Omega \times[0,1] \rightarrow F$ is said to be orientable if $\mathscr{S}(H)$ is nonempty. An orientation of $H$ is the choice of an equivalence class of $\mathscr{S}(H)$.

The following homotopy invariance property of the orientation of quasi-Fredholm maps is the analogue of Theorem 2.8. The proof is a straightforward consequence of Proposition 2.5.

Theorem 3.6. Let $H: \Omega \times[0,1] \rightarrow F$ be a homotopy of quasi-Fredholm maps. If a partial map $H_{\lambda}$ is oriented, then there exists and is unique an orientation of $H$ such that the orientation of $H_{\lambda}$ is inherited from that of $H$.

Let us now summarize the construction of the degree.

Definition 3.7. Let $f: \Omega \rightarrow F$ be an oriented quasi-Fredholm map and $U$ an open subset of $\Omega$. The triple $(f, U, 0)$ is said to be $q F$-admissible provided that $f^{-1}(0) \cap U$ is compact.

The construction of the degree for $q F$-admissible triples is in two steps. In the first one we consider triples $(f, U, 0)$ such that $f$ has a smoothing map $g$ with $(f-g)(U)$ contained in a finite dimensional subspace of $F$. In the second step we remove this assumption, defining the degree for all $q F$-admissible triples.

Step 3.8. Let $(f, U, 0)$ be a $q F$-admissible triple and let $g$ be a positively oriented smoothing map of $f$ such that $(f-g)(U)$ is contained in a finite dimensional subspace of $F$. 
As $f^{-1}(0) \cap U$ is compact, there exist a finite dimensional subspace $Z$ of $F$ and an open neighborhood $W$ of $f^{-1}(0)$ in $U$, such that $g$ is transverse to $Z$ in $W$. We may assume that $Z$ contains $(f-g)(U)$. Let $M=g^{-1}(Z) \cap W$. As seen at the end of Section 2, let $Z$ be oriented and orient $M$ in such a way that it is the oriented $\left.g\right|_{W}$-preimage of $Z$. One can easily verify that $\left(\left.f\right|_{M}\right)^{-1}(0)=f^{-1}(0) \cap U$. Thus $\left(\left.f\right|_{M}\right)^{-1}(0)$ is compact, and the Brouwer degree of the triple $\left(\left.f\right|_{M}, M, 0\right)$ turns out to be well defined.

Definition 3.9. Let $(f, U, 0)$ be a $q F$-admissible triple and let $g$ be a positively oriented smoothing map of $f$ such that $(f-g)(U)$ is contained in a finite dimensional subspace of $F$. Let $Z$ be a finite dimensional subspace of $F$ and $W$ an open neighborhood of $f^{-1}(0)$ in $U$ such that

(1) $Z$ contains $(f-g)(U)$,

(2) $g$ is transverse to $Z$ in $W$.

Assume $Z$ oriented and let $M$ be the oriented $\left.g\right|_{W}$-preimage of $Z$. Then, the degree of $(f, U, 0)$ is defined as

$$
\operatorname{deg}_{q F}(f, U, 0)=\operatorname{deg}_{B}\left(\left.f\right|_{M}, M, 0\right),
$$

where the right-hand side of the above formula is the Brouwer degree of the triple $\left(\left.f\right|_{M}\right.$, $M, 0)$.

In [4] it is proved that the above definition is well posed in the sense that the righthand side of (3.6) is independent of the choice of the smoothing map $g$, the open set $W$ and the subspace $Z$.

Step 3.10. Let us now extend the definition of degree to general $q F$-admissible triples.

Definition 3.11. Let $(f, U, 0)$ be a $q F$-admissible triple. Consider

(1) a positively oriented smoothing map $g$ of $f$;

(2) an open neighborhood $V$ of $f^{-1}(0) \cap U$ such that $\bar{V} \subseteq U, g$ is proper on $\bar{V}$ and $\left.(f-g)\right|_{\bar{V}}$ is compact;

(3) a continuous map $\xi: \bar{V} \rightarrow F$ having bounded finite dimensional image and such that

$$
\|g(x)-f(x)-\xi(x)\|<\rho, \quad \forall x \in \partial V
$$

where $\rho$ is the distance in $F$ between 0 and $f(\partial V)$.

Then,

$$
\operatorname{deg}_{q F}(f, U, 0)=\operatorname{deg}_{q F}(g-\xi, V, 0) .
$$

Observe that the right-hand side of (3.8) is well defined since the triple $(g-\xi, V, 0)$ is $q F$-admissible. Indeed, $g-\xi$ is proper on $\bar{V}$ and thus $(g-\xi)^{-1}(0)$ is a compact subset of $\bar{V}$ which is actually contained in $V$ by assumption (3).

In [4] it is proved that Definition 3.11 is well posed since formula (3.8) does not depend on $g, \xi$ and $V$.

We conclude the section by listing some properties of the degree. The proof of this result is in [4]. 
8 A degree theory for a class of perturbed Fredholm maps II

THEOREM 3.12. The following properties of the degree hold.

(1) (Normalization) Let $U$ be an open neighborhood of 0 in $E$ and let the identity I of $E$ be naturally oriented. Then,

$$
\operatorname{deg}_{q F}(I, U, 0)=1 .
$$

(2) (Additivity) Given a $q F$-admissible triple $(f, U, 0)$ and two disjoint open subsets $U_{1}$, $U_{2}$ of $U$ such that $f^{-1}(0) \cap U \subseteq U_{1} \cup U_{2}$, then

$$
\operatorname{deg}_{q F}(f, U, 0)=\operatorname{deg}_{q F}\left(f, U_{1}, 0\right)+\operatorname{deg}_{q F}\left(f, U_{2}, 0\right) .
$$

(3) (Excision) Given a qF-admissible triple $(f, U, 0)$ and an open subset $U_{1}$ of $U$ such that $f^{-1}(0) \cap U \subseteq U_{1}$, then

$$
\operatorname{deg}_{q F}(f, U, 0)=\operatorname{deg}_{q F}\left(f, U_{1}, 0\right)
$$

(4) (Existence) Given a $q F$-admissible triple $(f, U, 0)$, if

$$
\operatorname{deg}_{q F}(f, U, 0) \neq 0,
$$

then the equation $f(x)=0$ has a solution in $U$.

(5) (Homotopy invariance) Let $H: U \times[0,1] \rightarrow F$ be an oriented homotopy of quasiFredholm maps. If $H^{-1}(0)$ is compact, then $\operatorname{deg}_{q F}\left(H_{\lambda}, U, 0\right)$ is well defined and does not depend on $\lambda \in[0,1]$.

\section{Measures of noncompactness}

In this section we recall the definition and properties of the Kuratowski measure of noncompactness [13], together with some related concepts. For general reference, see, for example, Deimling [7].

From now on the spaces $E$ and $F$ are assumed to be infinite dimensional. As in the above section, $\Omega$ will stand for an open subset of $E$.

The Kuratowski measure of noncompactness $\alpha(A)$ of a bounded subset $A$ of $E$ is defined as the infimum of the real numbers $d>0$ such that $A$ admits a finite covering by sets of diameter less than $d$. If $A$ is unbounded, we set $\alpha(A)=+\infty$.

We summarize the following properties of the measure of noncompactness. Given a subset $A$ of $E$, we denote by $\overline{\operatorname{co}} A$ the closed convex hull of $A$, and by $[0,1] A$ the set

$$
\{\lambda x: \lambda \in[0,1], x \in A\} .
$$

Proposition 4.1. Let $A$ and $B$ be subsets of $E$. Then

(1) $\alpha(A)=0$ if and only if $\bar{A}$ is compact;

(2) $\alpha(\lambda A)=|\lambda| \alpha(A)$ for any $\lambda \in \mathbb{R}$;

(3) $\alpha(A+B) \leq \alpha(A)+\alpha(B)$;

(4) if $A \subseteq B$, then $\alpha(A) \leq \alpha(B)$;

(5) $\alpha(A \cup B)=\max \{\alpha(A), \alpha(B)\}$;

(6) $\alpha([0,1] A)=\alpha(A)$;

(7) $\alpha(\overline{\mathrm{CO}} A)=\alpha(A)$. 
Properties (1)-(6) are straightforward consequences of the definition, while the last one is due to Darbo [6].

Given a continuous map $f: \Omega \rightarrow F$, let $\alpha(f)$ and $\omega(f)$ be as in the introduction. It is important to observe that $\alpha(f)=0$ if and only if $f$ is completely continuous (i.e., the restriction of $f$ to any bounded subset of $\Omega$ is a compact map) and $\omega(f)>0$ only if $f$ is proper on bounded closed sets. For a complete list of properties of $\alpha(f)$ and $\omega(f)$ we refer to [12]. We need the following one concerning linear operators.

Proposition 4.2. Let $L: E \rightarrow F$ be a bounded linear operator. Then $\omega(L)>0$ if and only if $\operatorname{Im} L$ is closed and $\operatorname{dim} \operatorname{Ker} L<+\infty$.

As a consequence of Proposition 4.2 one gets that a bounded linear operator $L$ is Fredholm if and only if $\omega(L)>0$ and $\omega\left(L^{*}\right)>0$, where $L^{*}$ is the adjoint of $L$.

Let $f$ be as above and fix $p \in \Omega$. We recall the definitions of $\alpha_{p}(f)$ and $\omega_{p}(f)$ given in [5]. Let $B(p, s)$ denote the open ball in $E$ centered at $p$ with radius $s$. Suppose that $B(p, s) \subseteq \Omega$ and consider

$$
\alpha\left(\left.f\right|_{B(p, s)}\right)=\sup \left\{\frac{\alpha(f(A))}{\alpha(A)}: A \subseteq B(p, s), \alpha(A)>0\right\} .
$$

This is nondecreasing as a function of $s$. Hence, we can define

$$
\alpha_{p}(f)=\lim _{s \rightarrow 0} \alpha\left(\left.f\right|_{B(p, s)}\right)
$$

Clearly $\alpha_{p}(f) \leq \alpha(f)$ for any $p \in \Omega$. In an analogous way, we define

$$
\omega_{p}(f)=\lim _{s \rightarrow 0} \omega\left(\left.f\right|_{B(p, s)}\right),
$$

and we have $\omega_{p}(f) \geq \omega(f)$ for any $p$. It is easy to show that the main properties of $\alpha$ and $\omega$ hold, with minor changes, as well for $\alpha_{p}$ and $\omega_{p}$ (see [5]).

Proposition 4.3. Let $f: \Omega \rightarrow F$ be continuous and $p \in \Omega$. Then

(1) $\alpha_{p}(\lambda f)=|\lambda| \alpha_{p}(f)$ and $\omega_{p}(\lambda f)=|\lambda| \omega_{p}(f)$, for any $\lambda \in \mathbb{R}$;

(2) $\omega_{p}(f) \leq \alpha_{p}(f)$;

(3) $\left|\alpha_{p}(f)-\alpha_{p}(g)\right| \leq \alpha_{p}(f+g) \leq \alpha_{p}(f)+\alpha_{p}(g)$;

(4) $\omega_{p}(f)-\alpha_{p}(g) \leq \omega_{p}(f+g) \leq \omega_{p}(f)+\alpha_{p}(g)$;

(5) if $f$ is locally compact, $\alpha_{p}(f)=0$;

(6) if $\omega_{p}(f)>0, f$ is locally proper at $p$.

Clearly, for a bounded linear operator $L: E \rightarrow F$, the numbers $\alpha_{p}(L)$ and $\omega_{p}(L)$ do not depend on the point $p$ and coincide, respectively, with $\alpha(L)$ and $\omega(L)$. Furthermore, for the $C^{1}$ case we get the following result.

Proposition 4.4 ([5]). Let $f: \Omega \rightarrow F$ be of class $C^{1}$. Then, for any $p \in \Omega$ we have $\alpha_{p}(f)=$ $\alpha\left(f^{\prime}(p)\right)$ and $\omega_{p}(f)=\omega\left(f^{\prime}(p)\right)$.

Observe that if $f: \Omega \rightarrow F$ is a Fredholm map, as a straightforward consequence of Propositions 4.2 and 4.4 , we obtain $\omega_{p}(f)>0$ for any $p \in \Omega$. 
The following proposition extends to the continuous case an analogous result shown in [5] for $C^{1}$ maps.

Proposition 4.5. Let $g: \Omega \rightarrow F$ and $\sigma: \Omega \rightarrow \mathbb{R}$ be continuous. Consider the product map $f: \Omega \rightarrow F$ defined by $f(x)=\sigma(x) g(x)$. Then, for any $p \in \Omega$ we have $\alpha_{p}(f)=|\sigma(p)| \alpha_{p}(g)$ and $\omega_{p}(f)=|\sigma(p)| \omega_{p}(g)$.

Proof. Let $p \in \Omega$ be fixed, and assume first that $\sigma(p)=0$. Fix $\varepsilon>0$. As $\sigma$ is continuous, there exists $\bar{s}$ such that for any $s \leq \bar{s}$ and any $x \in B(p, s)$ one has $|\sigma(x)| \leq \varepsilon$ and, consequently, $f(x) \in[-\varepsilon, \varepsilon] g(x)$. It follows that $f(A) \subseteq[-\varepsilon, \varepsilon] g(A)$ for any $A \subseteq B(p, s)$. Hence, $\alpha(f(A)) \leq \varepsilon \alpha(g(A))$ for any $A \subseteq B(p, s)$, and this implies $\alpha\left(\left.f\right|_{B(p, s)}\right) \leq \varepsilon \alpha\left(\left.g\right|_{B(p, s)}\right)$. Taking the limit for $s \rightarrow 0$ we have $\alpha_{p}(f) \leq \varepsilon \alpha_{p}(g)$. Since $\varepsilon$ is arbitrary, we conclude that $\alpha_{p}(f)=0$.

In the general case, write

$$
f(x)=\sigma(p) g(x)+\tilde{f}(x)
$$

where $\tilde{f}(x)=\tilde{\sigma}(x) g(x)=(\sigma(x)-\sigma(p)) g(x)$. As $\tilde{\sigma}(p)=0$, we have $\alpha_{p}(\tilde{f})=0$. Therefore, by properties (1) and (3) in Proposition 4.3, we get $\alpha_{p}(f)=\alpha_{p}(\sigma(p) g)=|\sigma(p)| \alpha_{p}(g)$, as claimed. The case of $\omega_{p}(f)$ is analogous.

With an argument analogous to that used in [5], by means of Proposition 4.5 one can easily find examples of continuous maps $f$ such that $\alpha(f)=\infty$ and $\alpha_{p}(f)<\infty$ for any $p$, and examples of continuous maps $f$ with $\omega(f)=0$ and $\omega_{p}(f)>0$ for any $p$. Moreover, in [5] there is an example of a map $f$ such that $\alpha(f)>0$ and $\alpha_{p}(f)=0$ for any $p$.

In the sequel we will consider also maps $G$ defined on the product space $E \times \mathbb{R}$. In order to define $\alpha_{(p, \lambda)}(G)$, we consider the norm

$$
\|(p, \lambda)\|=\max \{\|p\|,|\lambda|\} .
$$

The natural projection of $E \times \mathbb{R}$ onto the first factor will be denoted by $\pi_{1}$.

Remark 4.6. With the above norm, $\pi_{1}$ is nonexpansive. Therefore $\alpha\left(\pi_{1}(X)\right) \leq \alpha(X)$ for any subset $X$ of $E \times \mathbb{R}$. More precisely, since $\mathbb{R}$ is finite dimensional, if $X \subseteq E \times \mathbb{R}$ is bounded, we have $\alpha\left(\pi_{1}(X)\right)=\alpha(X)$.

We conclude the section with the following technical result, which is a straightforward consequence of Proposition 4.5 and which will be useful in the construction of the degree for weakly $\alpha$-Fredholm maps (see Section 6 below).

Corollary 4.7. Given a continuous map $\varphi: \Omega \rightarrow F$, consider the map

$$
\Phi: \Omega \times[0,1] \longrightarrow F, \quad \Phi(x, \lambda)=\lambda \varphi(x) .
$$

Then, for any fixed pair $(p, \lambda) \in \Omega \times[0,1]$ we have

$$
\alpha_{(p, \lambda)}(\Phi)=\lambda \alpha_{p}(\varphi)
$$

Proof. Apply Proposition 4.5 and observe that, given $p \in \Omega$ and $\lambda \in[0,1]$, one has $\alpha_{(p, \lambda)}(\varphi)=\alpha_{p}(\varphi)$. 


\section{Degree for $\alpha$-Fredholm maps}

In this section we sketch the construction of the degree for $\alpha$-Fredholm maps introduced in [1]. These maps are special noncompact perturbations of Fredholm maps, defined in terms of the numbers $\alpha_{p}$ and $\omega_{p}$. Precisely, an $\alpha$-Fredholm map $f: \Omega \rightarrow F$ is of the form $f=g-k$, where $g$ is a Fredholm map of index zero, $k$ is a continuous map and $\alpha_{p}(k)<\omega_{p}(g)$ for every $p$.

The degree is given as an integer valued map defined on a class of triples that we will call admissible $\alpha$-Fredholm triples. This class is recalled in the following two definitions.

Definition 5.1. Let $g: \Omega \rightarrow F$ be a Fredholm map of index zero, $k: \Omega \rightarrow F$ a continuous map, and $U$ an open subset of $\Omega$. The triple $(g, U, k)$ is said to be $\alpha$-Fredholm if for any $p \in U$ we have

$$
\alpha_{p}(k)<\omega_{p}(g)
$$

Definition 5.2. An $\alpha$-Fredholm triple $(g, U, k)$ is said to be admissible if

(i) $g$ is oriented;

(ii) the solution set $S=\{x \in U: g(x)=k(x)\}$ is compact.

Definition 5.3. Let $(g, U, k)$ be an admissible $\alpha$-Fredholm triple and

$$
\mathscr{V}=\left\{V_{1}, \ldots, V_{N}\right\}
$$

a finite covering of open balls of its solution set $S$. We say that $\mathscr{V}$ is an $\alpha$-covering of $S$ (relative to $(g, U, k))$ if for any $i \in\{1, \ldots, N\}$ the following properties hold:

(i) the ball $\tilde{V}_{i}$ of double radius and same center as $V_{i}$ is contained in $U$;

(ii) $\alpha\left(\left.k\right|_{\tilde{V}_{i}}\right)<\omega\left(\left.g\right|_{\tilde{V}_{i}}\right)$.

Let $(g, U, k)$ be an admissible $\alpha$-Fredholm triple and $\mathscr{V}=\left\{V_{1}, \ldots, V_{N}\right\}$ an $\alpha$-covering of the solution set $S$. We define the following sequence $\left\{C_{n}\right\}$ of convex closed subsets of E:

$$
C_{1}=\overline{\mathrm{co}}\left(\bigcup_{i=1}^{N}\left\{x \in V_{i}: g(x) \in k\left(\tilde{V}_{i}\right)\right\}\right)
$$

and, inductively,

$$
C_{n}=\overline{\operatorname{co}}\left(\bigcup_{i=1}^{N}\left\{x \in V_{i}: g(x) \in k\left(\tilde{V}_{i} \cap C_{n-1}\right)\right\}\right), \quad n \geq 2 .
$$

Observe that, by induction, $C_{n+1} \subseteq C_{n}$ and $S \subseteq C_{n}$ for any $n \geq 1$. Then the set

$$
C_{\infty}=\bigcap_{n \geq 1} C_{n}
$$

turns out to be closed, convex, and containing $S$. Consequently, if $S$ is nonempty, so is $C_{\infty}$. To emphasize the fact that the set $C_{\infty}$ is uniquely determined by the covering $\mathscr{V}$, 
sometimes it will be denoted by $C_{\infty}^{\mathcal{V}}$. In addition $C_{\infty}$ verifies the following two properties (see [1] for the proof):

(1) $\left\{x \in V_{i}: g(x) \in k\left(\tilde{V}_{i} \cap C_{\infty}\right)\right\} \subseteq C_{\infty}$, for any $i=1, \ldots, N$;

(2) $C_{\infty}$ is compact.

Definition 5.4. Let $(g, U, k)$ be an admissible $\alpha$-Fredholm triple, $\mathscr{V}=\left\{V_{1}, \ldots, V_{N}\right\}$ an $\alpha$ covering of the solution set $S$, and $C$ a compact convex set. We say that $(\mathscr{V}, C)$ is an $\alpha$-pair (relative to $(g, U, k))$ if the following properties hold:

(1) $U \cap C \neq \varnothing$;

(2) $C_{\infty} \subseteq C$;

(3) $\left\{x \in V_{i}: g(x) \in k\left(\tilde{V}_{i} \cap C\right)\right\} \subseteq C$ for any $i=1, \ldots, N$.

Remark 5.5. Given any admissible $\alpha$-Fredholm triple $(g, U, k)$, it is always possible to find an $\alpha$-pair $(\mathscr{V}, C)$. Indeed, assume that the solution set $S$ is nonempty. Then, given any $\alpha$ covering $\mathscr{V}$ of $S$, the corresponding compact set $C_{\infty}^{\mathscr{V}}$ is nonempty as well and, clearly, the pair $\left(\mathscr{V}, C_{\infty}^{\mathscr{V}}\right)$ verifies properties (1)-(3) in Definition 5.4. If, on the other hand, $S=\varnothing$, one can check that $(\{\varnothing\},\{p\})$ is an $\alpha$-pair for any $p \in U$.

Let $(g, U, k)$ be an admissible $\alpha$-Fredholm triple and let $(\mathscr{V}, C)$ be an $\alpha$-pair. Consider a retraction $r: E \rightarrow C$, whose existence is ensured by Dugundji's Extension theorem [8]. Denote $V=\bigcup_{i=1}^{N} V_{i}$, where $\left\{V_{1}, \ldots, V_{N}\right\}=\mathscr{V}$, and let $W$ be a (possibly empty) open subset of $V$ containing $S$ such that, for any $i, x \in W \cap V_{i}$ implies $r(x) \in \widetilde{V}_{i}$. For example, if $\rho$ denotes the minimum of the radii of the balls $V_{i}$, one may take as $W$ the set

$$
\{x \in V:\|x-r(x)\|<\rho\} .
$$

Observe that property (3) above implies that the two equations $g(x)=k(x)$ and $g(x)=$ $k(r(x))$ have the same solution set in $W$ (notice that the composition $k r$ is defined in the open set $r^{-1}(U)$ containing $W$ ). The map $k r$ is locally compact (even if not necessarily compact), hence the triple $(g-k r, W, 0)$ is $q F$-admissible (recall Definition 3.7$)$. We define the degree of the triple $(g, U, k), \operatorname{deg}_{*}(g, U, k)$ in symbols, as follows:

$$
\operatorname{deg}_{*}(g, U, k)=\operatorname{deg}_{q F}(g-k r, W, 0)
$$

where the right-hand side is the degree defined in Section 3.

The following definition summarizes the above construction.

Definition 5.6. Let $(g, U, k)$ be an admissible $\alpha$-Fredholm triple and $(\mathscr{V}, C)$ an $\alpha$-pair. Consider a retraction $r: E \rightarrow C$. Denote $V=\bigcup_{i=1}^{N} V_{i}$, where $\left\{V_{1}, \ldots, V_{N}\right\}=\mathscr{V}$. Let $W$ be an open subset of $V$ containing $S$ such that, for any $i, x \in W \cap V_{i}$ implies $r(x) \in \widetilde{V}_{i}$. We set

$$
\operatorname{deg}_{*}(g, U, k)=\operatorname{deg}_{q F}(g-k r, W, 0)
$$

As proved in [1], the above definition is well posed since the right-hand side of formula (5.8) is independent of the choice of the $\alpha$-pair $(\mathscr{V}, C)$, of the retraction $r$ and of the open set $W$. 
We conclude this section by stating the most important properties of the degree. Actually, in [1] only the fundamental properties (i.e., normalization, additivity and homotopy invariance) were stated and proved. The excision and existence properties are easy consequences of the additivity.

THeOREM 5.7. The following properties hold.

(1) (Normalization) Let the identity I of E be naturally oriented. Then

$$
\operatorname{deg}_{*}(I, E, 0)=1 .
$$

(2) (Additivity) Given an admissible $\alpha$-Fredholm triple $(g, U, k)$ and two disjoint open subsets $U_{1}, U_{2}$ of $U$, assume that $S=\{x \in U: g(x)=k(x)\}$ is contained in $U_{1} \cup U_{2}$. Then

$$
\operatorname{deg}_{*}(g, U, k)=\operatorname{deg}_{*}\left(g, U_{1}, k\right)+\operatorname{deg}_{*}\left(g, U_{2}, k\right) .
$$

(3) (Excision) Given an admissible $\alpha$-Fredholm triple $(g, U, k)$ and an open subset $U_{1}$ of $U$, assume that $S$ is contained in $U_{1}$. Then

$$
\operatorname{deg}_{*}(g, U, k)=\operatorname{deg}_{*}\left(g, U_{1}, k\right) .
$$

(4) (Existence) Given an admissible $\alpha$-Fredholm triple $(g, U, k)$, if

$$
\operatorname{deg}_{*}(g, U, k) \neq 0,
$$

then the equation $g(x)=k(x)$ has a solution in $U$.

(5) (Homotopy invariance) Let $H: U \times[0,1] \rightarrow F$ be a homotopy of the form $H(x, \lambda)=$ $G(x, \lambda)-K(x, \lambda)$, where $G$ is of class $C^{1}$, any $G_{\lambda}=G(\cdot, \lambda)$ is Fredholm of index zero, $K$ is continuous, and $\alpha_{(p, \lambda)}(K)<\omega_{(p, \lambda)}(G)$ for any pair $(p, \lambda) \in U \times[0,1]$. Assume that $G$ is oriented and that $H^{-1}(0)$ is compact. Then $\operatorname{deg}_{*}\left(G_{\lambda}, U, K_{\lambda}\right)$ is well defined and independent of $\lambda \in[0,1]$.

\section{Degree for weakly $\alpha$-Fredholm maps}

We present here an extension of the degree for $\alpha$-Fredholm maps to a more general class of maps, called weakly $\alpha$-Fredholm. These are of the form $f=g-k: \Omega \rightarrow F$, where $g$ is Fredholm of index zero, $k$ is continuous and the following condition is verified: for any $p \in \Omega$ there exists $s>0$ such that for any $A \subseteq B(p, s)$ with $\alpha(A)>0$ we have $\alpha(k(A))<$ $\omega_{p}(g) \alpha(A)$.

The reader can verify that $\alpha$-Fredholm maps are also weakly $\alpha$-Fredholm.

As in the previous section, this degree is an integer valued map defined on a special class of triples, called admissible weakly $\alpha$-Fredholm.

Definition 6.1. Let $g: \Omega \rightarrow F$ be a Fredholm map of index zero, $k: \Omega \rightarrow F$ a continuous map and $U$ an open subset of $\Omega$. The triple $(g, U, k)$ is said to be weakly $\alpha$-Fredholm if for any $p \in U$ there exists $s>0$ such that for any $A \subseteq B(p, s)$ with $\alpha(A)>0$ we have

$$
\alpha(k(A))<\omega_{p}(g) \alpha(A)
$$


14 A degree theory for a class of perturbed Fredholm maps II

Let $(g, U, k)$ be a weakly $\alpha$-Fredholm triple. As a consequence of Definition 6.1, given $p \in U$ there exists $s>0$ such that

$$
\alpha(k(A))<\alpha(g(A)), \quad \text { for any } A \subseteq B(p, s) \text { with } \alpha(A)>0 .
$$

Thus, any compact subset of $U$ admits a neighborhood as in the following definition.

Definition 6.2. Let $(g, U, k)$ be a weakly $\alpha$-Fredholm triple, and $Q$ a compact subset of $U$. An open neighborhood $V$ of $Q$ is said to be an $\alpha$-neighborhood of $Q$ (relative to $(g, U, k)$ ) if the following properties hold:

(i) $\bar{V} \subseteq U$ and $k(\bar{V})$ is bounded;

(ii) $\alpha(k(A))<\alpha(g(A))$, for any $A \subseteq \bar{V}$ with $\alpha(A)>0$.

Lemma 6.3. Let $(g, U, k)$ be a weakly $\alpha$-Fredholm triple, $Q$ a compact subset of $U$, and $V$ an $\alpha$-neighborhood of $Q$ (relative to $(g, U, k)$ ). Then, the homotopy

$$
\Psi: \bar{V} \times[0,1] \longrightarrow F, \quad \Psi(x, \lambda)=g(x)-\lambda k(x)
$$

is proper.

Proof. Let $C \subseteq F$ be compact. We need to show that the set $D=\Psi^{-1}(C)$ is compact. As in Section 4 , let $\pi_{1}$ denote the natural projection of $E \times \mathbb{R}$ onto the first factor. Notice that, given $x \in \pi_{1}(D)$, we have $g(x) \in C+[0,1] k(x)$. Thus,

$$
g\left(\pi_{1}(D)\right) \subseteq C+[0,1] k\left(\pi_{1}(D)\right) .
$$

Consequently, by the properties of the measure of noncompactness,

$$
\alpha\left(g\left(\pi_{1}(D)\right)\right) \leq \alpha(C)+\alpha\left(k\left(\pi_{1}(D)\right)\right)=\alpha\left(k\left(\pi_{1}(D)\right)\right) .
$$

As $V$ is an $\alpha$-neighborhood of $Q$, property (ii) in Definition 6.2 implies $\alpha\left(\pi_{1}(D)\right)=0$. Moreover, by Remark 4.6, since $D \subseteq \pi_{1}(D) \times[0,1]$ we have

$$
\alpha(D) \leq \alpha\left(\pi_{1}(D) \times[0,1]\right)=\alpha\left(\pi_{1}(D)\right)=0 .
$$

Hence, $\alpha(D)=0$. Therefore $D$ is compact, being closed in $E \times[0,1]$ (recall Proposition 4.1).

As a consequence of this result we deduce the following property.

Corollary 6.4. Let $\Psi$ be as in Lemma 6.3. Then, any partial map $\Psi(\cdot, \lambda)$ is proper on $\bar{V}$.

We introduce now the concept of admissible weakly $\alpha$-Fredholm triple.

Definition 6.5. A weakly $\alpha$-Fredholm triple $(g, U, k)$ is said to be admissible if

(i) $g$ is oriented;

(ii) the solution set $S=\{x \in U: g(x)=k(x)\}$ is compact.

Given an admissible weakly $\alpha$-Fredholm triple $(g, U, k)$ and an $\alpha$-neighborhood $V$ of $S$, let us show that, for $\varepsilon>0$ sufficiently small, $(g, V,(1-\varepsilon) k)$ is an admissible $\alpha$-Fredholm 
triple. To see this observe first that, by Definition 6.1, $\alpha_{p}(k) \leq \omega_{p}(g)$ for any $p \in U$. Therefore, for any $p \in U$ and any positive $\varepsilon<1$ we have

$$
\alpha_{p}((1-\varepsilon) k)=(1-\varepsilon) \alpha_{p}(k)<\omega_{p}(g)
$$

and, consequently, $(g, U,(1-\varepsilon) k)$ is an $\alpha$-Fredholm triple. We claim that, for $\varepsilon>0$ small, this triple is admissible (i.e., $S_{\varepsilon}=\{x \in V: g(x)=(1-\varepsilon) k(x)\}$ is compact). Observe that, by Corollary 6.4 , the map $g-k$ is proper on $\bar{V}$. Thus, the number

$$
\delta=\inf \{\|g(x)-k(x)\|: x \in \partial V\}
$$

is positive. Moreover, set

$$
\gamma=\sup \{\|k(x)\|: x \in \partial V\}
$$

As $k(\bar{V})$ is bounded, it follows that $\gamma$ is finite. Now, given $x \in \partial V$ and $\varepsilon<\min \{1, \delta / \gamma\}$ (we put $\delta / \gamma=+\infty$ if $\gamma=0$ ), we have

$$
\|g(x)-(1-\varepsilon) k(x)\| \geq\|g(x)-k(x)\|-\varepsilon\|k(x)\| \geq \delta-\varepsilon \gamma>0
$$

and, consequently, the equation $g(x)=(1-\varepsilon) k(x)$ has no solutions on $\partial V$. Since, by Corollary 6.4, the map $g-(1-\varepsilon) k$ is proper on $\bar{V}$, it follows that $S_{\varepsilon}$ is compact. Hence, $(g, V,(1-\varepsilon) k)$ is an admissible $\alpha$-Fredholm triple. This argument suggests the following definition.

Definition 6.6. Let $(g, U, k)$ be an admissible weakly $\alpha$-Fredholm triple, and $V$ an $\alpha$ neighborhood of the solution set $S$. Put $\delta$ and $\gamma$ as in (6.8) and (6.9). If $0<\varepsilon<\min \{1, \delta / \gamma\}$, we set

$$
\operatorname{deg}(g, U, k)=\operatorname{deg}_{*}(g, V,(1-\varepsilon) k) .
$$

The next proposition shows that the above definition is well posed.

Proposition 6.7. Let $(g, U, k)$ be an admissible weakly $\alpha$-Fredholm triple, and let $V_{1}$ and $V_{2}$ be two $\alpha$-neighborhoods of the solution set $S$. Put

$$
\delta_{i}=\inf \left\{\|g(x)-k(x)\|: x \in \partial V_{i}\right\}, \quad \gamma_{i}=\sup \left\{\|k(x)\|: x \in \partial V_{i}\right\}, \quad i=1,2 .
$$

If $0<\varepsilon_{i}<\min \left\{1, \delta_{i} / \gamma_{i}\right\}$, for $i=1,2$, then

$$
\operatorname{deg}_{*}\left(g, V_{1},\left(1-\varepsilon_{1}\right) k\right)=\operatorname{deg}_{*}\left(g, V_{2},\left(1-\varepsilon_{2}\right) k\right) .
$$

Proof. Since the intersection of two $\alpha$-neighborhoods of $S$ is still an $\alpha$-neighborhood, without loss of generality we can assume that $V_{1} \supseteq V_{2}$. Set

$$
\delta_{3}=\inf \left\{\|g(x)-k(x)\|: x \in \bar{V}_{1} \backslash V_{2}\right\}, \quad \gamma_{3}=\sup \left\{\|k(x)\|: x \in \bar{V}_{1} \backslash V_{2}\right\},
$$

and fix a positive $\varepsilon_{3}<\min \left\{\varepsilon_{1}, \varepsilon_{2}, \delta_{3} / \gamma_{3}\right\}$. We claim that

$$
\operatorname{deg}_{*}\left(g, V_{i},\left(1-\varepsilon_{i}\right) k\right)=\operatorname{deg}_{*}\left(g, V_{i},\left(1-\varepsilon_{3}\right) k\right), \quad i=1,2 .
$$


16 A degree theory for a class of perturbed Fredholm maps II

To see this, consider the homotopy

$$
\begin{gathered}
H: \bar{V}_{1} \times[0,1] \longrightarrow F, \\
H(x, \lambda)=g(x)-\left(1-(1-\lambda) \varepsilon_{1}-\lambda \varepsilon_{3}\right) k(x) .
\end{gathered}
$$

We have $H(x, \lambda)=G(x, \lambda)-K(x, \lambda)$, where

$$
G(x, \lambda)=g(x), \quad K(x, \lambda)=\left(1-(1-\lambda) \varepsilon_{1}-\lambda \varepsilon_{3}\right) k(x) .
$$

Hence, by Corollary 4.7, for any fixed $(p, \lambda) \in \bar{V}_{1} \times[0,1]$ we have

$$
\alpha_{(p, \lambda)}(K)=\left(1-(1-\lambda) \varepsilon_{1}-\lambda \varepsilon_{3}\right) \alpha_{p}(k)<\omega_{p}(g)=\omega_{(p, \lambda)}(G) .
$$

Moreover, given $x \in \partial V_{1}$ and $\lambda \in[0,1]$, we have

$$
\|G(x, \lambda)-K(x, \lambda)\| \geq\|g(x)-k(x)\|-\left((1-\lambda) \varepsilon_{1}+\lambda \varepsilon_{3}\right)\|k(x)\| \geq \delta_{1}-\varepsilon_{1} \gamma_{1}>0 .
$$

Since, by Lemma 6.3, the map $H$ is proper, from the latter inequality it follows that the solution set $\left\{(x, \lambda) \in V_{1} \times[0,1]: H(x, \lambda)=0\right\}$ is compact. Hence, we can apply the homotopy invariance property of the degree for $\alpha$-Fredholm triples, and we have

$$
\operatorname{deg}_{*}\left(g, V_{1},\left(1-\varepsilon_{1}\right) k\right)=\operatorname{deg}_{*}\left(g, V_{1},\left(1-\varepsilon_{3}\right) k\right) .
$$

In an analogous way, we have

$$
\operatorname{deg}_{*}\left(g, V_{2},\left(1-\varepsilon_{2}\right) k\right)=\operatorname{deg}_{*}\left(g, V_{2},\left(1-\varepsilon_{3}\right) k\right),
$$

as claimed.

Now, given $x \in \bar{V}_{1} \backslash V_{2}$, we have

$$
\left\|g(x)-\left(1-\varepsilon_{3}\right) k(x)\right\| \geq\|g(x)-k(x)\|-\varepsilon_{3}\|k(x)\| \geq \delta_{3}-\varepsilon_{3} \gamma_{3}>0 .
$$

Therefore, we can apply the excision property of the degree for $\alpha$-Fredholm triples, obtaining

$$
\operatorname{deg}_{*}\left(g, V_{1},\left(1-\varepsilon_{3}\right) k\right)=\operatorname{deg}_{*}\left(g, V_{2},\left(1-\varepsilon_{3}\right) k\right),
$$

and the assertion follows.

\section{Properties of the degree}

We start this section by introducing the concept of weakly $\alpha$-Fredholm homotopy. Given $\lambda \in[0,1]$ and $\sigma>0$, we denote $I_{\sigma}=(\lambda-\sigma, \lambda+\sigma) \cap[0,1]$.

Definition 7.1. Let $\Omega \subseteq E$ be open, and $H: \Omega \times[0,1] \rightarrow F$ a continuous map of the form

$$
H(x, \lambda)=G(x, \lambda)-K(x, \lambda) .
$$


We say that $H$ a weakly $\alpha$-Fredholm homotopy if $G$ is $C^{1}$, any $G_{\lambda}$ is Fredholm of index zero, and for any pair $(p, \lambda) \in \Omega \times[0,1]$ there exist $s, \sigma>0$ such that for any $D \subseteq B(p, s) \times I_{\sigma}$ with $\alpha(D)>0$ we have

$$
\alpha(K(D))<\omega_{(p, \lambda)}(G) \alpha(D)
$$

TheOREM 7.2. The following properties of the degree hold.

(1) (Normalization) Let the identity I of E be naturally oriented. Then

$$
\operatorname{deg}(I, E, 0)=1
$$

(2) (Additivity) Given an admissible weakly $\alpha$-Fredholm triple $(g, U, k)$ and two disjoint open subsets $U_{1}, U_{2}$ of $U$, assume that $S=\{x \in U: g(x)=k(x)\}$ is contained in $U_{1} \cup U_{2}$. Then

$$
\operatorname{deg}(g, U, k)=\operatorname{deg}\left(g, U_{1}, k\right)+\operatorname{deg}\left(g, U_{2}, k\right)
$$

(3) (Homotopy invariance) Let $H: U \times[0,1] \rightarrow F$ be a weakly $\alpha$-Fredholm homotopy of the form $H(x, \lambda)=G(x, \lambda)-K(x, \lambda)$. Assume that $G$ is oriented and that $H^{-1}(0)$ is compact. Then $\operatorname{deg}\left(G_{\lambda}, U, K_{\lambda}\right)$ is well defined and does not depend on $\lambda \in[0,1]$.

Proof. (1) (Normalization) It coincides with the normalization property of the degree for admissible $\alpha$-Fredholm triples (and of course for $q F$-admissible triples).

(2) (Additivity) Let $S_{1}=S \cap U_{1}$ and $S_{2}=S \cap U_{2}$, so that $S=S_{1} \cup S_{2}$. Clearly $S_{1}$ and $S_{2}$ are compact and, consequently, the triples $\left(g, U_{1}, k\right)$ and $\left(g, U_{2}, k\right)$ are admissible.

Let $V$ be an $\alpha$-neighborhood of $S$ relative to $(g, U, k)$, and let $V_{1}=V \cap U_{1}$ and $V_{2}=$ $V \cap U_{2}$. Clearly, $V_{1}$ and $V_{2}$ are two disjoint $\alpha$-neighborhoods of $S_{1}$ and $S_{2}$ relative to $\left(g, U_{1}, k\right)$ and $\left(g, U_{2}, k\right)$, respectively. By Definition 6.6, choosing $\varepsilon>0$ sufficiently small we have

$$
\begin{gathered}
\operatorname{deg}(g, U, k)=\operatorname{deg}_{*}(g, V,(1-\varepsilon) k), \\
\operatorname{deg}\left(g, U_{i}, k\right)=\operatorname{deg}_{*}\left(g, V_{i},(1-\varepsilon) k\right), \quad i=1,2 .
\end{gathered}
$$

On the other hand, the additivity property of the degree for $\alpha$-Fredholm triples implies

$$
\operatorname{deg}_{*}(g, V,(1-\varepsilon) k)=\operatorname{deg}_{*}\left(g, V_{1},(1-\varepsilon) k\right)+\operatorname{deg}_{*}\left(g, V_{2},(1-\varepsilon) k\right),
$$

and the assertion follows.

(3) (Homotopy invariance) For $\lambda \in[0,1]$, let $\Sigma_{\lambda}$ denote the set $\left\{x \in U: G_{\lambda}(x)=K_{\lambda}(x)\right\}$. Given any $\lambda$, the fact that $\left(G_{\lambda}, U, K_{\lambda}\right)$ is an admissible weakly $\alpha$-Fredholm triple follows easily from the compactness of $\Sigma_{\lambda}$ and observing that $\omega_{p}\left(G_{\lambda}\right) \geq \omega_{(p, \lambda)}(G)$ for any $p \in U$.

To verify that the property holds, it is sufficient to show that the integer valued function

$$
\lambda \longmapsto \operatorname{deg}\left(G_{\lambda}, U, K_{\lambda}\right)
$$


is locally constant. To this purpose, fix $\tau \in[0,1]$ and, given $\rho>0$, denote $J_{\rho}=[\tau-\rho, \tau+$ $\rho] \cap[0,1]$. It is possible to find $\rho>0$ and an open subset $V$ of $U$ with the following properties:

(i) $V$ contains $\Sigma_{\lambda}$ for any $\lambda \in J_{\rho}$;

(ii) $\bar{V} \subseteq U$ and $K\left(\bar{V} \times J_{\rho}\right)$ is bounded;

(iii) $\alpha(K(D))<\alpha(G(D))$, for any $D \subseteq \bar{V} \times J_{\rho}$ with $\alpha(D)>0$.

In particular, $V$ is an $\alpha$-neighborhood of $\Sigma_{\lambda}$ relative to $\left(G_{\lambda}, U, K_{\lambda}\right)$ for any $\lambda \in J_{\rho}$.

Consider the map

$$
\hat{\Psi}: \bar{V} \times J_{\rho} \times[0,1] \longrightarrow F, \quad \hat{\Psi}(x, \lambda, \mu)=G(x, \lambda)-\mu K(x, \lambda) .
$$

Using an argument analogous to the proof of Lemma 6.3, one can show that $\hat{\Psi}$ is proper. Now, let $\lambda \in J_{\rho}$ be fixed. Set

$$
\delta_{\lambda}=\inf \left\{\left\|G_{\lambda}(x)-K_{\lambda}(x)\right\|: x \in \partial V\right\}, \quad \gamma_{\lambda}=\sup \left\{\left\|K_{\lambda}(x)\right\|: x \in \partial V\right\}
$$

and, analogously,

$$
\begin{gathered}
\delta=\inf \left\{\|G(x, \lambda)-K(x, \lambda)\|: x \in \partial V \times J_{\rho}\right\}, \\
\gamma=\sup \left\{\|K(x, \lambda)\|: x \in \partial V \times J_{\rho}\right\} .
\end{gathered}
$$

Fix a positive $\varepsilon<\min \{1, \delta / \gamma\}$. As $\delta \leq \delta_{\lambda}$ and $\gamma \geq \gamma_{\lambda}$, it follows that $\varepsilon<\delta_{\lambda} / \gamma_{\lambda}$. Consequently, by Definition 6.6, we have

$$
\operatorname{deg}\left(G_{\lambda}, U, K_{\lambda}\right)=\operatorname{deg}_{*}\left(G_{\lambda}, V,(1-\varepsilon) K_{\lambda}\right) .
$$

Now, consider the following homotopy:

$$
\hat{H}: \bar{V} \times J_{\rho} \longrightarrow F, \quad \hat{H}(x, \lambda)=G(x, \lambda)-(1-\varepsilon) K(x, \lambda) .
$$

Notice that for any fixed pair $(p, \lambda) \in \bar{V} \times J_{\rho}$ we have

$$
\alpha_{(p, \lambda)}((1-\varepsilon) K)<\omega_{(p, \lambda)}(G) .
$$

Moreover, $\hat{H}$ is proper since it coincides with the partial map $\hat{\Psi}(\cdot, \cdot, 1-\varepsilon)$. As the equation $\hat{H}(x, \lambda)=0$ has no solutions on $\partial V \times J_{\rho}$, it follows that $\hat{H}^{-1}(0)$ is a compact subset of $V \times J_{\rho}$. Therefore, the homotopy invariance property of the degree for $\alpha$-Fredholm triples implies that $\operatorname{deg}_{*}\left(G_{\lambda}, V,(1-\varepsilon) K_{\lambda}\right)$ does not depend on $\lambda \in J_{\rho}$. Hence, $\operatorname{deg}\left(G_{\lambda}, U, K_{\lambda}\right)$ is independent of $\lambda \in J_{\rho}$, and this completes the proof.

\section{Comparison with the Nussbaum degree for local $\alpha$-condensing vector fields}

The purpose of this section is to show that, in a sense to be specified, our concept of degree extends the degree for local $\alpha$-condensing perturbations of the identity, introduced by Nussbaum in $[14,15]$.

Let $f: \Omega \rightarrow F$ be a continuous map. We recall the following definitions. The map $f$ is said to be $\alpha$-contractive if $\alpha(f(A)) \leq \mu \alpha(A)$ for some $\mu<1$ and any $A \subseteq \Omega$. The map $f$ 
is said to be $\alpha$-condensing if $\alpha(f(A))<\alpha(A)$ for any $A \subseteq \Omega$ such that $0<\alpha(A)<+\infty$. If for any $p \in \Omega$ there exists a neighborhood $V_{p}$ of $p$ such that $\left.f\right|_{V_{p}}$ is $\alpha$-contractive (resp., $\alpha$-condensing), $f$ is said to be local $\alpha$-contractive (resp., local $\alpha$-condensing).

In $[14,15]$, Nussbaum developed a degree theory for triples of the form $(I-k, U, 0)$, where $k$ is local $\alpha$-condensing. Precisely, let $U$ be an open subset of $\Omega$ and $k: \Omega \rightarrow E$ a local $\alpha$-condensing map. Assume that the set $S=\{x \in U:(I-k)(x)=0\}$ is compact. Then, the triple $(I-k, U, 0)$ is admissible for the Nussbaum degree ( $N$-admissible, for short). We will denote by $\operatorname{deg}_{N}(I-k, U, 0)$ the Nussbaum degree of an $N$-admissible triple.

Let $(I-k, U, 0)$ be an $N$-admissible triple. According to Definition $6.5,(I, U, k)$ is an admissible weakly $\alpha$-Fredholm triple provided that $I$ is oriented. We claim that, if we assign the natural orientation to $I$, it follows that

$$
\operatorname{deg}(I, U, k)=\operatorname{deg}_{N}(I-k, U, 0) .
$$

Indeed, let $V$ be an $\alpha$-neighborhood of $S$ relative to $(I, U, k)$. By the excision property of the Nussbaum degree we have

$$
\operatorname{deg}_{N}(I-k, U, 0)=\operatorname{deg}_{N}(I-k, V, 0) .
$$

Now, if $\varepsilon>0$ is sufficiently small we have

$$
\operatorname{deg}_{N}(I-k, V, 0)=\operatorname{deg}_{N}(I-(1-\varepsilon) k, V, 0)
$$

by the definition of the Nussbaum degree, and

$$
\operatorname{deg}(I, U, k)=\operatorname{deg}_{*}(I, V,(1-\varepsilon) k)
$$

by Definition 6.6. The claim now follows from the fact that the degree for $\alpha$-Fredholm triples and the Nussbaum degree coincide on the class of local $\alpha$-contractive vector fields, provided that the identity is naturally oriented (see [1]).

\section{References}

[1] P. Benevieri, A. Calamai, and M. Furi, A degree theory for a class of perturbed Fredholm maps, Fixed Point Theory \& Applications 2005 (2005), no. 2, 185-206.

[2] P. Benevieri and M. Furi, A simple notion of orientability for Fredholm maps of index zero between Banach manifolds and degree theory, Annales des Sciences Mathématiques du Québec 22 (1998), no. 2, 131-148.

[3] __ On the concept of orientability for Fredholm maps between real Banach manifolds, Topological Methods in Nonlinear Analysis 16 (2000), no. 2, 279-306.

[4] - A Degree theory for locally compact perturbations of Fredholm maps in Banach spaces, to appear in Abstract and Applied Analysis.

[5] A. Calamai, The invariance of domain theorem for compact perturbations of nonlinear Fredholm maps of index zero, Nonlinear Functional Analysis and Applications 9 (2004), no. 2, 185-194.

[6] G. Darbo, Punti uniti in trasformazioni a codominio non compatto, Rendiconti del Seminario Matematico della Università di Padova 24 (1955), 84-92.

[7] K. Deimling, Nonlinear Functional Analysis, Springer, Berlin, 1985.

[8] J. Dugundji, An extension of Tietze's theorem, Pacific Journal of Mathematics 1 (1951), no. 3, 353-367. 


\section{A degree theory for a class of perturbed Fredholm maps II}

[9] D. E. Edmunds and J. R. L. Webb, Remarks on nonlinear spectral theory, Unione Matematica Italiana. Bollettino. B. Serie 62 (1983), no. 1, 377-390.

[10] M. A. Efendiev, On a new variant degree theory of mapping and its application, World Congress of Nonlinear Analysts '92, Vol. I-IV (Tampa, Fla, 1992), de Gruyter, Berlin, 1996, pp. 111-123.

[11] M. A. Efendiev and W. L. Wendland, Nonlinear Riemann-Hilbert problems for multiply connected domains, Nonlinear Analysis 27 (1996), no. 1, 37-58.

[12] M. Furi, M. Martelli, and A. Vignoli, Contributions to the spectral theory for nonlinear operators in Banach spaces, Annali di Matematica Pura ed Applicata. Serie Quarta 118 (1978), 229-294.

[13] C. Kuratowski, Topologie. Vol. I, 4th ed., Monografie Matematyczne, vol. 20, Państwowe Wydawnictwo Naukowe, Warsaw, 1958.

[14] R. D. Nussbaum, The fixed point index for local condensing maps, Annali di Matematica Pura ed Applicata. Serie Quarta 89 (1971), 217-258.

[15] Degree theory for local condensing maps, Journal of Mathematical Analysis and Applications 37 (1972), no. 3, 741-766.

[16] V. G. Zvyagin and N. M. Ratiner, Oriented degree of Fredholm maps of nonnegative index and its application to global bifurcation of solutions, Global Analysis-Studies and Applications, V, Lecture Notes in Math., vol. 1520, Springer, Berlin, 1992, pp. 111-137.

Pierluigi Benevieri: Dipartimento di Matematica Applicata “G. Sansone," Via S. Marta 3, 50139 Firenze, Italy

E-mail address: pierluigi.benevieri@unifi.it

Alessandro Calamai: Dipartimento di Matematica “U. Dini,” Viale G.B. Morgagni 67/A, 50134 Firenze, Italy

E-mail address: calamai@math.unifi.it

Massimo Furi: Dipartimento di Matematica Applicata “G. Sansone," Via S. Marta 3, 50139 Firenze, Italy

E-mail address: massimo.furi@unifi.it 